\title{
Deslocamento miscível de nitrato e fosfato proveniente de água residuária da suinocultura em colunas de solo ${ }^{1}$
}

\author{
Marcelo H. Anami ${ }^{2}$, Silvio C. Sampaio ${ }^{2}$, Morgana Suszek2 ${ }^{2}$, Simone D. Gomes ${ }^{2} \&$ Manoel M. F. de Queiroz ${ }^{2}$
}

\begin{abstract}
RESUMO
A fertirrigação com águas residuárias da suinocultura vem sendo muito difundida no País, principalmente na região sul do Brasil, entretanto, o alto potencial poluidor dos dejetos pode tornar-se uma ameaça de contaminação de solos e águas superficiais e subterrâneas se utilizados em quantidades excessivas. Neste trabalho o objetivo principal foi avaliar o processo de lixiviação de íons nitrato e fosfato em colunas de solo, obtendo-se os coeficientes de dispersão hidrodinâmico e o fator de retardamento para a determinação do potencial de contaminação dos lençóis de água subterrâneos. Verificou-se, ainda, o efeito da aplicação de águas residuárias da suinocultura tratada com reatores anaeróbios sobre as propriedades físico-químicas do solo, cujos resultados indicaram que o potencial de contaminação dos lençóis de água subterrâneos pelo íon nitrato é elevado, ao contrário do que ocorre com o íon fosfato, que apresentou baixo potencial de contaminação em função da sua alta reatividade.
\end{abstract}

Palavras-chave: movimento de íons, fertirrigação, contaminação do solo

\section{Miscible displacement of nitrate and phosphate from swine wastewater in soil columns}

\begin{abstract}
Fertigation with wastewater from swine is very much used, mainly in the southern region of Brazil. However, the high polluting potential of these wastewaters represents a threat of soil contamination of surface and underground waters if used in excessive amounts. The objective of this work was to evaluate the leaching process of nitrate and phosphate ions in soil columns, getting the hydrodynamic dispersion coefficient and factor of retardation and potential of contamination of underground water. The effect of wastewater application on physical and chemical properties of the soil was verified. The results showed that the potential for contamination of underground water by nitrate ions is high, in contrast to what occurs with phosphate ions that presented low potential of contamination due to their high reactivity.
\end{abstract}

Key words: ion movement, fertigation, soil pollution

1 Parte da dissertação de mestrado do primeiro autor, apresentada à UNIOESTE

2 RHESA/UNIOESTE, Rua Universitária 2069, CEP 85819-110. Cascavel, PR. Fone: (45) 3220-3262. E-mail: ssampaio@unioeste.br; mhanami@bol.com.br; morgana_eq@hotmail.com; simoned@unioeste.br; mfqueiroz@unioeste.br 


\section{INTRODUÇÃO}

É grande o potencial de utilização de águas residuárias provenientes das práticas suinícolas na agricultura, passível de proporcionar economia e ganhos financeiros com o aumento do rendimento das culturas, redução da aplicação de fertilizantes químicos e consumo de água e a melhoria das condições físicas, químicas e biológicas do solo, porém já são relatados problemas de contaminação do solo, rios e cursos d'água no Sul do Brasil devido ao uso excessivo de dejetos suínos sem tratamento ou tratados de forma inadequada (Costa et al., 2000).

Burkart \& Stoner (2002) constataram que a criação de gado confinado juntamente com a permeabilidade do solo, aumenta a contaminação das águas subterrâneas localmente, com aumento do nitrogênio $\left(\mathrm{NO}_{3}-\mathrm{N}\right)$; este fato pode ocorrer com os dejetos de suínos em função da semelhança orgânica dos materiais. Gliessman (2001) considera, como um dos fatores de sustentabilidade dos agroecossistemas, a qualidade da água subterrânea, em particular a lixiviação de nutrientes, como nitratos e outros contaminantes.

Nos sistemas de tratamento de efluentes em lagoas, a ocorrência de fósforo está na forma de íons fosfato e, apesar deste elemento ficar retido facilmente na superfície e sub-superfície do solo, devido a mudanças químicas e adsorção, ainda pode ser um poluente das águas subterrâneas ocasionado pelo processo de lixiviação do elemento, que permanece livre na solução do solo (Canter et al., 1987).

As maiores dificuldades para a determinação de modelos de remoção de íons no solo estão relacionadas à grande variabilidade espacial e temporal da condutividade hidráulica do solo; à variabilidade da adsorção do íon no solo; à falta de informações sobre o efeito da água residuária devido aos conteúdos de alumínio, ferro e cálcio (que formam complexos) e à falta de dados dos níveis de acidez e/ou alcalinidade no solo; com isto, torna-se necessário o conhecimento do ponto de carga zero, que é definido como o pH onde existe máxima agregação das partículas, possibilitando a formação da estrutura do solo, fator este importante em solos argilosos de carga variável, visto que o conhecimento da estrutura do solo é fundamental para se entender uma das propriedades do solo, que é a permeabilidade, a qual interfere no fluxo de líquidos através de um meio poroso (Cardoso et al., 2001).

A avaliação do transporte de solutos pode ser feita por modelos numéricos e físicos; os modelos físicos a destacar, são: colunas de solo (modelo unidimensional); aparato de Hele-Shaw (bidimensional) e tanque de areia (tridimensional); referidos modelos são submetidos a remoção ou injeção de água ou, ainda, a uma carga contaminada, e a resposta aos modelos é obtida através de medições diretas da concentração do soluto, no início e durante o deslocamento do fluido através da coluna (Canter et al., 1987).

Objetivou-se, através do presente estudo, avaliar o deslocamento miscível de nitrato e fosfato proveniente de águas residuárias da suinocultura tratadas em reator anaeróbio em colunas de solo, com a determinação dos parâmetros do modelo dispersivo-convectivo, os efeitos físico-químicos no solo e o ponto de carga zero.

\section{MATERIAL E MÉTODOS}

O trabalho foi conduzido em duas etapas; na primeira, realizou-se a aplicação da água residuária da suinocultura em 6 colunas de solo, avaliando-se o deslocamento miscível de nitrato e fosfato, sendo calculados, com os dados obtidos experimentalmente, os coeficientes de dispersão hidrodinâmico e fator de retardamento; efetuou-se, ainda, o ajuste de curvas de concentração relativa dos íons de nitrato e fosfato, com a utilização do software CXTFIT (Toride et al., 1999) e, na segunda etapa, avaliou-se o efeito da aplicação da água residuária sobre os parâmetros físico e químicos do solo e se determinou o ponto de carga zero.

\section{Primeira etapa}

A água residuária da suinocultura utilizada no experimento proveio de uma lagoa de algas, integrante de um sistema de tratamento de dejetos suíno, denominado biossistema integrado, composto de um biodigestor, tanque de sedimentação, tanque de algas e tanque de peixes.

Os parâmetros físicos, químicos e biológicos da água residuária, se encontram na Tabela 1.

Tabela 1. Parâmetros da água residuária da suinocultura utilizada no experimento

\begin{tabular}{lcr}
\hline Parâmetro & Unidade & Valor \\
$\mathrm{pH}$ & - & 7,77 \\
Condutividade elétrica & $\mathrm{mS} \mathrm{cm}^{-1}$ & 10,12 \\
Nitrato & $\mathrm{mg} \mathrm{L}^{-1}$ & 8,00 \\
Nitrogênio total & $\mathrm{mg} \mathrm{L}^{-1}$ & 147,95 \\
Fósforo total & $\mathrm{mg} \mathrm{L}^{-1}$ & 30,80 \\
Fosfato & $\mathrm{mg} \mathrm{L}^{-1}$ & 20,99 \\
Sódio & $\mathrm{mg} \mathrm{L}^{-1}$ & 315,00 \\
Potássio & $\mathrm{mg} \mathrm{L}^{-1}$ & 450,00 \\
DQ0 & $\mathrm{mg} \mathrm{L}^{-1}$ & 2062,00 \\
DB0/5 & $\mathrm{mg} \mathrm{L}^{-1}$ & 594,00 \\
Coliformes totais & $\mathrm{NMP} 100 \mathrm{~mL}^{-1}$ & 90000 \\
Coliformes termotolerantes & $\mathrm{NMP} 100 \mathrm{~mL}^{-1}$ & 50000 \\
\hline
\end{tabular}

As colunas em PVC utilizadas no experimento mediam $7,5 \mathrm{~cm}$ de diâmetro e $70 \mathrm{~cm}$ de comprimento, com $50 \mathrm{~cm}$ de altura de solo; a carga hidráulca acima do solo foi mantida em $10 \mathrm{~cm}$.

Seis colunas em um suporte de madeira foram montadas, sendo aplicada primeiro água destilada, a partir de frascos de Mariotte para saturação do meio poroso das colunas; após atingida a saturação, realizou-se a aplicação da água residuária da suinocultura.

O solo, classificado como Latossolo Vermelho Distroférrico Típico (EMBRAPA, 1999), foi coletado em profundidade de aproximadamente três metros para simular uma situação de fundo de lagoa anaeróbia, enquanto os parâmetros físicos e químicos do solo foram obtidos conforme metodologia da EMBRAPA (1997) e se acham na Tabela 2.

O solo foi acondicionado nas colunas, de forma a se obter uma densidade constante em todas elas, conforme os parâmetros físicos apresentados na Tabela 3. 
Tabela 2. Parâmetros físicos e químicos do solo utilizado nas colunas

\begin{tabular}{lcr}
\hline Parâmetro & Unidade & Valor \\
pH $\left(\mathrm{CaCl}_{2}\right)$ & - & 4,21 \\
Fósforo & $\mathrm{mg} \mathrm{dm}^{-3}$ & 1,22 \\
Carbono & $\mathrm{g} \mathrm{dm}^{-3}$ & 0,58 \\
Potássio & $\mathrm{cmol}_{\mathrm{C}} \mathrm{dm}^{-3}$ & 0,03 \\
Cálcio & $\mathrm{cmol}_{\mathrm{C}} \mathrm{dm}^{-3}$ & 1,49 \\
Magnésio & $\mathrm{cmol}_{\mathrm{C} \mathrm{dm}} \mathrm{dm}^{-3}$ & 0,23 \\
$\mathrm{H}+$ Al & $\mathrm{cmol}_{\mathrm{C} \mathrm{dm}} \mathrm{dm}_{\mathrm{C}}^{-3}$ & 6,14 \\
Alumínio & $\mathrm{cmol}_{\mathrm{C} \mathrm{dm}} \mathrm{dm}^{-3}$ & 0,68 \\
Cobre & $\mathrm{mg} \mathrm{dm}^{-3}$ & 5,19 \\
Zinco & $\mathrm{mg} \mathrm{dm}^{-3}$ & 0,34 \\
Ferro & $\mathrm{mg} \mathrm{dm}^{-3}$ & 116,75 \\
Manganês & $\mathrm{mg} \mathrm{dm}^{-3}$ & 65,08 \\
Argila & $\%$ & 79,00 \\
Silte & $\%$ & 10,00 \\
Areia & $\%$ & 11,00 \\
Soma de Bases & $\%$ & 1,75 \\
CTC & $\mathrm{cmol}_{\mathrm{C} \mathrm{dm}}^{-3}$ & 7,89 \\
V & $\mathrm{cmol}_{\mathrm{C} \mathrm{dm}}^{-3}$ & 22,19 \\
\hline
\end{tabular}

Tabela 3. Parâmetros físicos das colunas utilizadas no experimento

\begin{tabular}{lcc}
\hline Coluna de solo & Unidade & Valor \\
Massa total de solo & $\mathrm{Kg}$ & 1,97 \\
Volume de poros & $\mathrm{m}^{3}$ & 0,001503 \\
Volume de solo na coluna & $\mathrm{m}^{3}$ & 0,002209 \\
Porosidade & $\%$ & 68,03 \\
Velocidade real & $\mathrm{m} \mathrm{dia}^{-1}$ & 10,0056 \\
Densidade de fluxo & $\mathrm{m}^{3} \mathrm{~m}^{-2} \mathrm{dia}^{-1}$ & 8,23104 \\
Condutividade hidráulica & $\mathrm{m} \mathrm{dia}^{-1}$ & 6,8592 \\
Densidade do solo & $\mathrm{g} \mathrm{cm}^{3}$ & 0,892 \\
Umidade residual & $\mathrm{cm}^{-3}$ & 0,196 \\
Umidade saturada & $\mathrm{cm}^{-3}$ & 0,6803 \\
\hline
\end{tabular}

A quantidade de água residuária aplicada em cada coluna, de modo a determinar as curvas de eluição, foi quatro vezes o volume de poros para o íon nitrato (Ferreira \& Martinez, 1997) e para o íon fosfato (Zhao et al., 2000).

Coletaram-se todas as frações do efluente lixiviado das colunas representativas do volume de poros correspondente, sendo analisados nitrato, fosfato e condutividade elétrica, de acordo com metodologia descrita por Eaton \& Clesceri (1995); entretanto, somente algumas das amostras dos volumes de poros foram separadas para análise de nitrato e fosfato, determinados a partir da análise de condutividade elétrica, devido ao elevado custo para a análise de todas as amostras coletadas.

Para a construção das curvas de eluição e determinação do coeficiente de dispersão e fator de retardamento para o nitrato e fosfato, utilizou-se o programa computacional CXTFIT (Toride et al., 1999).

\section{Segunda etapa}

Nesta etapa se avaliaram os efeitos decorrentes da aplicação da água residuária sobre as propriedades físico-químicas do solo, de acordo com metodologia descrita por EMBRAPA (1997).
Os dados obtidos foram submetidos a análise de variância e teste de comparação de médias, utilizando-se o Teste de Tukey ao nível de 5\% de significância.

$\mathrm{O}$ ponto de carga zero, também conhecido por $\mathrm{pH}_{0}$, foi definido pelo método sugerido por Uehara \& Gillman (1981) apud Cardoso et al. (2001).

\section{RESULTADOS E DISCUSSÃO}

Os resultados apresentados na Figura 1 demonstram tendência de aumento da condutividade elétrica com o aumento do volume de fluido lixiviado, fato este também verificado para o nitrato e fosfato, confirmando a relação existente entre a condutividade elétrica e os teores dos elementos analisados.

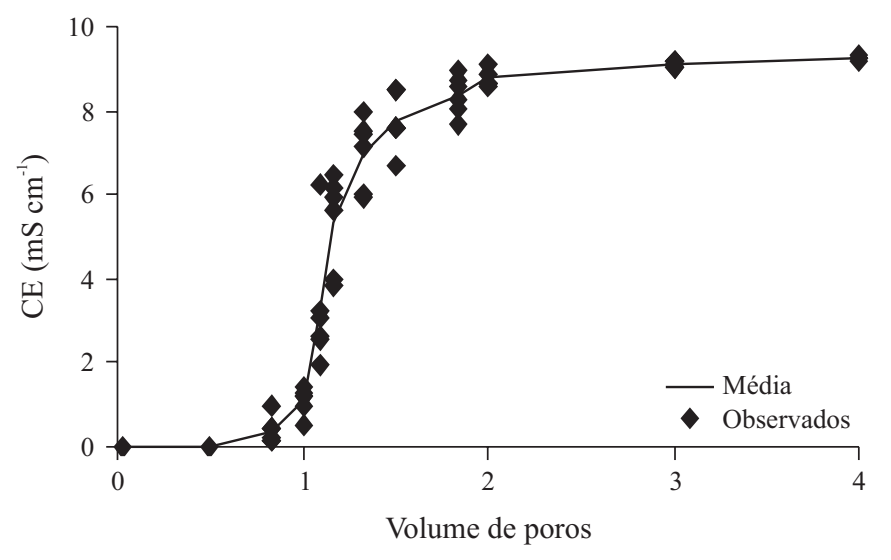

Figura 1. Valores médios e observados de condutividade elétrica do efluente lixiviado em relação ao volume de poros

Os resultados obtidos pelo programa CXTFIT para o nitrato: Concentração inicial $=8,0 \mathrm{mg} \mathrm{L}^{-1}$, Coeficiente de dispersão $=0,11168$, Fator de retardamento $=0,87372$ e $\mathrm{R}^{2}=$ 0,9742, mostraram alta correlação entre os valores observados experimentalmente e os valores ajustados. Os resultados de fator de retardamento próximos a 1 indicam baixa interação solo-soluto, conforme observado por Correa et al. (1999).

O ajuste da curva de eluição para o nitrato (Figura 2A) indica que a partir de dois volumes de poros ocorre estabilização da concentração relativa, demonstrando que o elemento tem comportamento não reativo, conforme observado por Bohn (1985) apud Feigin et al. (1991).

Schwartz et al. (1999), estudando as influências dos distúrbios antes e depois da formulação das condições de contorno para estimativa dos coeficientes de dispersão e fator de retardamento em coluna de solo curtas utilizando íon bromo, encontraram valores de coeficiente de dispersão de 0,684 a $0,883\left(\mathrm{~m}^{2} \mathrm{dia}^{-1}\right)$ e fator de retardamento de 1,09 a 2,02 tendo, como influência para essas variações, o tipo de solo e o horizonte para coleta das amostras da coluna, mostrando que o nitrato tem comportamento semelhante ao íon bromo. 


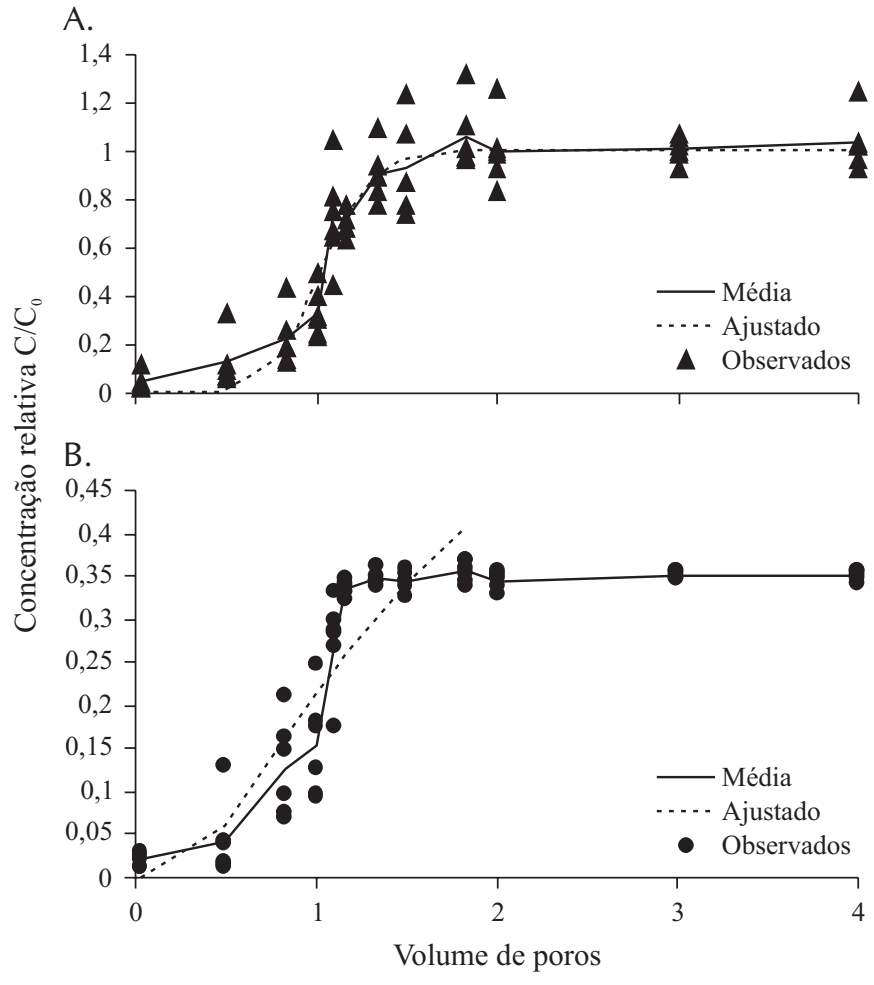

Figura 2. Curva de eluição para nitrato (2A) e fosfato (2B)

Gomes et al. (2004) estudando a lixiviação de nitrato em colunas de solo argiloso e arenoso, com e sem presença de calcário, utilizando como fluido deslocador água residuária de frigorífico, observaram que a aplicação de calcário pode aumentar o deslocamento de nitrato não só para solo argiloso mas, também, para solo arenoso. $\mathrm{O}$ ajuste das curvas através do programa CXTFIT entre os valores observados para o ensaio, produziu coeficientes de correlação acima de $80 \%$.

Os resultados obtidos pelo Programa CXTFIT para o elemento fosfato: Concentração inicial $=20,99 \mathrm{mg} \mathrm{L}^{-1}$, Coeficiente de dispersão $=6,31808$, Fator de retardamento $=$ 4,40801 e $R^{2}=0,8849$, apresentaram alta correlação entre os valores observados experimentalmente em relação aos valores ajustados, enquanto os de fator de retardamento acima de 2 ou 3 indicam alta interação solo-soluto, demonstrando que o íon fosfato é altamente reativo (Jury et al., 1991).

Núñez-Delgado et al. (2002) constataram fatores de retardamento para o íon fosfato, maiores que 5, para todos os tipos de solo após a passagem de 5 volumes de poros.

$\mathrm{O}$ ajuste da curva de eluição para o fosfato (Figura 2B) demonstra haver, ainda, tendência de elevação em 2 volumes de poros chegando à concentração relativa $C / C_{0}$ de 0,43; a mesma observação foi feita por Bohn (1985) apud Feigin et al. (1991) com a diferença de que o aumento da concentração relativa começou a partir de 8 volumes de poros chegando ao valor de 0,5 a partir de 14 volumes de poros.

Os resultados obtidos podem ser explicados pela alta correlação entre as superfícies específica de óxidos de ferro e adsorção de fósforo em solos argilosos, conforme Fontes \&
Weed (1996); outro fator que pode ter influenciado na obtenção desses dados pode estar relacionado ao fato da possibilidade de ter ocorrido fluxo preferencial, ainda que o valor da umidade imóvel em relação à umidade total $\left(\theta_{\mathrm{im}} / \theta\right)$ tenha o valor de 0,2881 , abaixo da faixa de 0,48 a 0,82 definido por Lee et al. (2001) como indicativo de considerável fluxo preferencial.

A redução dos teores de fosfato está associada à capacidade de adsorção específica por unidade de área superficial para um grupo selecionado de amostras de solo, com uma mineralogia variada na composição, em termos de óxidos de ferro (Fontes \& Weed, 1996).

Os resultados obtidos no experimento tendem a indicar que novos trabalhos envolvendo a dinâmica do fosfato proveniente da águas residuárias devem ser desenvolvidos.

A partir dos resultados da análise de solo antes e depois da aplicação das águas residuárias apresentados na Tabela 4, observa-se uma melhora significativa nas propriedades químicas, considerando-se a fertilidade do solo.

Tabela 4. Características fisico-químicas do solo, antes e após aplicação de água residuária

\begin{tabular}{|c|c|c|c|}
\hline Parâmetro & Unidade & $\begin{array}{c}\text { Valor } \\
\text { (antes da aplicação) }\end{array}$ & $\begin{array}{c}\text { Valor } \\
\text { (após a aplicação) }\end{array}$ \\
\hline $\mathrm{pH}\left(\mathrm{CaCl}_{2}\right)$ & & $4,21 \mathrm{a}$ & $6,98 \mathrm{~b}$ \\
\hline Fósforo & $\mathrm{mg} \mathrm{dm}^{-3}$ & $1,22 \mathrm{a}$ & $227,53 \mathrm{~b}$ \\
\hline Carbono & $\mathrm{g} \mathrm{dm}^{-3}$ & $0,58 \mathrm{a}$ & $2,55 \mathrm{~b}$ \\
\hline Potássio & $\mathrm{cmol} \mathrm{dm}^{-3}$ & $0,03 \mathrm{a}$ & $0,86 \mathrm{~b}$ \\
\hline Cálcio & $\mathrm{cmol} \mathrm{dm} \mathrm{dm}^{-3}$ & $1,49 \mathrm{a}$ & $4,40 \mathrm{~b}$ \\
\hline Magnésio & $\mathrm{cmol} \mathrm{dm}{ }^{-3}$ & $0,23 \mathrm{a}$ & $0,74 \mathrm{~b}$ \\
\hline $\mathrm{H}+\mathrm{Al}$ & $\mathrm{cmol} \mathrm{dm}^{-3}$ & $6,14 \mathrm{a}$ & $2,20 \mathrm{~b}$ \\
\hline Alumínio & $\mathrm{cmol} \mathrm{dm}^{-3}$ & $0,68 \mathrm{a}$ & $0,00 \mathrm{~b}$ \\
\hline Cobre & $\mathrm{mg} \mathrm{dm}^{-3}$ & $5,19 \mathrm{a}$ & $9,10 \mathrm{~b}$ \\
\hline Zinco & $\mathrm{mg} \mathrm{dm} \mathrm{m}^{-3}$ & $0,34 \mathrm{a}$ & $5,10 \mathrm{~b}$ \\
\hline Ferro & $\mathrm{mg} \mathrm{dm} \mathrm{m}^{-3}$ & $116,75 \mathrm{a}$ & $61,00 \mathrm{~b}$ \\
\hline Manganês & $\mathrm{mg} \mathrm{dm}{ }^{-3}$ & $65,08 \mathrm{a}$ & $119,17 \mathrm{~b}$ \\
\hline Argila & $\%$ & $79,00 \mathrm{a}$ & $79,00 \mathrm{a}$ \\
\hline Silte & $\%$ & $10,00 \mathrm{a}$ & $10,00 \mathrm{a}$ \\
\hline Areia & $\%$ & $11,00 \mathrm{a}$ & $11,00 \mathrm{a}$ \\
\hline Soma de Bases & $\mathrm{cmol} \mathrm{dm}^{-3}$ & $1,75 \mathrm{a}$ & $6,00 \mathrm{~b}$ \\
\hline CTC & $\mathrm{cmol} \mathrm{dm}^{-3}$ & $7,89 \mathrm{a}$ & $8,20 \mathrm{a}$ \\
\hline V & $\%$ & $22,19 \mathrm{a}$ & $73,17 \mathrm{~b}$ \\
\hline
\end{tabular}

* Letras iguais na mesma linha não diferem entre si pelo Teste de Tukey ao nível de $5 \%$ de significância

Os valores apresentados na análise química do solo se mostram altamente favoráveis ao condicionamento do solo, confirmando o observado por Choudhary et al. (1996).

As reduções dos teores de ferro e alumínio no solo, além de estarem relacionadas à interação com a matéria orgânica (que teve aumento significativo para 4\%), mostram ser esta relação provável com o complexo que o fósforo forma com esses metais, tornando-os insolúveis (Hodson et al., 2001).

O valor de carbono obtido com a aplicação de água residuária (2,55 $\left.\mathrm{g} \mathrm{dm}^{-3}\right)$ permaneceu próximo do valor obtido por Martin Neto et al. (1996), que foi de $2,7 \mathrm{~g} \mathrm{dm}^{-3}$, para área nativa (sem cultivo ou revolvimento no horizonte $\mathrm{A}_{11}$ ). 
Notou-se redução do ponto de carga zero $(3,94)$, considerando-se o inicial e a média das profundidades $(3,43)$ após a aplicação das águas residuárias e também o aumento do pH do solo, conforme a Tabela 5; este evento pode estar relacionado a dois fatores: ao fato de que o fosfato é um ânion orgânico grande e confere carga negativa da mistura, reduzindo o ponto de carga zero, e ao aumento do teor de matéria orgânica que, mesmo em baixas concentrações, altera o ponto de carga zero em função dos radicais da matéria orgânica do grupo funcional carboxílico (dissociado) promoverem consumo de $\mathrm{H}^{+}$elevando o $\mathrm{pH}$ e aumentando as cargas negativas reduzindo, conseqüentemente, o ponto de carga zero.

Tabela 5. Ponto de carga zero (PCZ) do solo antes e após aplicação de água residuária

\begin{tabular}{cccc}
\hline Aplicação de água residuária & Profundidade (cm) & PCZ & pH \\
Antes & - & 3,94 & 4,20 \\
\hline \multirow{3}{*}{ Após } & $0-15$ & 3,30 & 6,98 \\
& $15-30$ & 4,00 & 6,98 \\
& $30-50$ & 3,00 & 6,98 \\
& Média das profundidades & 3,43 & 6,98 \\
\hline
\end{tabular}

A redução do ponto de carga zero pode ser atribuída ao fosfato que fica adsorvido ao solo, como se observa na Figura 2B, aumentando a adsorção de cátions (Stoop, 1980); o cálcio adsorvido interfere na determinação do ponto de carga zero, exceto que este seja percolado na forma de cloreto de cálcio, o que não foi avaliado no experimento.

\section{CONCLUSÕES}

1. O íon nitrato da água residuária da suinocultura apresentou elevado potencial de contaminação de solos e águas subterrâneas, visto seu rápido deslocamento no perfil do solo demonstrado pelo coeficiente de dispersão hidrodinâmico $(0,112)$ e fator de retardamento $(0,874)$ encontrados.

2. Para o íon fosfato, a possibilidade de poluição é menor se comparada com o nitrato, em virtude do coeficiente de dispersão $(6,318)$ e fator de retardamento $(4,408)$ mais elevado, demonstrando sua reatividade com o solo.

3. A aplicação da água residuária da suinocultura no solo promoveu melhorias nas suas características físico-químicas e redução no ponto de carga zero.

\section{LITERATURA CITADA}

Burkart, M. R.; Stoner, J. D. Nitrate in aquifers beneath agricultural systems. Water Science and Technology, London, v.45, n.9, p.19-29, 2002.

Canter, L. W.; Knox, R. C.; Fairchild, D. M. Ground water quality protection. Florida: Lewis Publishers, 1987. 256p.

Cardoso, D. L.; Gentelini, D. P.; Larios, M. R. B. Uma metodologia para a estimativa do ponto de carga zero em solos. In: Congresso Brasileiro de Engenharia Agrícola, 30, 2001, Foz do Iguaçu. Anais... Foz do Iguaçu: SBEA, 2001. CD Rom.
Choudhary, M.; Bailey, L. D.; Grant, C. A. Review of the use of swine manure in crop production: effects on yield and composition and on soil and water quality. Waste Management and Reserch, Copenhagen, v.14, p.581-595, 1996.

Correa, M. M.; Lima, L. A.; Martinez, M. A.; Riginato, R. L. O.; Sampaio, S. C. Deslocamento miscível de sulfona de aldicarbe em colunas de solo. Engenharia Agrícola e Ambiental, Campina Grande, v.3, n.2, p.217-221, 1999.

Costa, R. H. R.; Bavaresco, A. S. L.; Medri, W.; Philippi, L. S. Tertiary treatment of piggery waste in water hyacinth ponds. Water Science and Technology, London, v.42, n.1011, p.211-214, 2000.

Eaton, A. D.; Clesceri, L. S.; Standard methods for the examination of water and wastewater. 19 th. Washington: AWWA/ APHA, 1995.

EMBRAPA - Empresa Brasileira de Pesquisa Agropecuária. Centro Nacional de Pesquisa de Solos. Manual de métodos de análise de solo. Rio de janeiro: Embrapa Solos, 1997, 212p.

EMBRAPA - Empresa Brasileira de Pesquisa Agropecuária. Centro Nacional de Pesquisa de Solos. Sistema brasileiro de classificação de solos. Rio de Janeiro: Embrapa Solos, 1999, 412p.

Feigin, A.; Ravina, I.; Shalhevet, J. Irrigation whit treated sewage effluent, management for environmental protection: Advanced series in agricultural sciences. Berlin: Springer-Verlang, 1991, 17p.

Ferreira, P. A.; Martinez, M. A. Movimento e modelagem de sais no solo, In: Gheyi, H. R.; Queiroz, J. E.; Medeiros, J. F. de. Manejo e controle de salinidade na agricultura irrigada. Campina Grande: UFPB/SBEA, 1997, p.171-206.

Fontes, M. P. F.; Weed, S. B. Phosphate adsorption by clays from Brazilian oxisols relationships with specific surface area and mineralogy. Geoderma, Amsterdam, v.72, p.37-51, 1996.

Gliessman, S. R. Agroecologia: Processos ecológicos em agricultura sustentável. Porto Alegre: UFRGS, 2001, 653p.

Gomes, E. R. S.; Sampaio, S. C.; Corrêa, M. M.; Vilas Boas, M. A.; Alves, L. F. A.; Sobrinho, T. A. Movimento de nitrato proveniente de águas residuárias em colunas de solo. Revista Engenharia Agrícola, Jaboticabal, v.24, n.3, p.557-568, 2004.

Hodson, M. E.; valsami-Jones, E.; Cotter-Howells, J. D.; Dubbin, W. E.; Kemp, A. J.; Thornton, I.; Waren, A. Effect of bone meal (Calcium phosphate) amendments on metal release from contaminated soils - a leaching column study. Environmental Pollution, London, v.12, p.233-243, 2001.

Jury, W.; Gardner, W. R.; Gardner, W. H. Soil physics. New York: John Wiley \& Sons INC., 1991, 328p.

Lee, J.; Horton, H.; Noborio, K.; Jaynes, D. B. Caracterization of preferencial flow in undisturbed, structured soil column using vertical TDR Probe. Journal of Contaminant Hydrology, Amsterdam, v.51, p.131-144. 2001.

Martin Neto, L.; Andriulo, A.; Traghetta, D. G. Fracionamento físico da matéria orgânica do solo e caracterização por espectroscopia de EPR. São Carlos: Embrapa Infromações Agropecuárias, 1996, 7p. Comunicado Técnico.

Núñez-Delgado, A.; López-Periago, E.; Diaz-Fierros, F. Pollution attenuation by soil receiving cattle slurry after passage of slurry-like feed solution: Column Experiments. Bioresource Technology, United Kingdom, v.84, p.229236, 2002. 
Schawartz, R. C.; Mcinnes, K. J.; Juo, A. S. R.; Wilding, L. P. Boundary effects on solute transport in finite soil columns. Water Resources Research, Washington, v.35, n.3, p.671681, 1999.

Stoop, W. A. Íon adsortion mchanisms in oxidic solil, implications for point of zero charge determinations, Geoderma, Amsterdam, v.23, p.303-314, 1980.
Toride, N.; Leij, F. J.; van Genuchten M. The CXTFIT code for estimating transport parameters from laboratory or field tracer experiments, versión 2.1. California: Agricultural Research Service, USDA, 1999. 148p.

Zhao, J. Q; Li, Y. C.; Norland, M. R. Leading potencial of nitrogen and phosphorus in substrates of soil disposal mounds in Florida's everglades. Journal of Soil Contamination, v.9, n.2, p.119-132, 2000. 